\title{
Mitochondrial D-loop informative SNPs in identification of dog's breed
}

\author{
ANGELIKA TKACZYK, KRZYSZTOF KOWAL, BRYGIDA ŚLASKA \\ Institute of Biological Bases of Animal Production, Faculty of Animal Sciences and Bioeconomy, \\ University of Life Sciences in Lublin, Akademicka 13, 20-950 Lublin, Poland
}

Tkaczyk A., Kowal K., Ślaska B.

\section{Mitochondrial D-loop informative SNPs in identification of dog's breed}

Summary

The aim of the study was to determine differences at the molecular level between different breeds of dogs on the basis of a comparative analysis of the mtDNA D-loop sequence. The material used in the study consisted of blood collected from 40 Doberman Pinscher, Dachshund, German Shepherd, and Crossbreed dogs. The investigations involved DNA extraction, amplification and sequencing of the mtDNA D-loop, as well as bioinformatic analyses. Fifteen SNP (single nucleotide polymorphism) mutations were identified. This study proposes a new solution for dog breed identification, i.e. the use of SNPs specific to each breed. The analysis revealed the presence of already known SNPs in various configurations depending on the breed. Breed-specific SNP patterns were identified in $94 \%$ of the Doberman dogs, and two SNP patterns were found in $73 \%$ of the German Shepherd dogs. This subject requires further research. However, given the limited amount of data on the use of mtDNA in determination of breed affiliation, the present results seem to be promising.

Keywords: mtDNA, SNP, Dachshund, Doberman, German Shepherd

Biological material from domestic dogs, i.e. tissues, hair, blood, and droppings, is increasingly collected and secured at incident sites and constitutes important evidence in criminal and civil proceedings (2, 3 ). It is not always possible to employ the analysis of STR (short tandem repeats) markers specific for the domestic dog, because of the degree of degradation of the material (3). Similarly, the use of comparative morphological analysis of hair material can sometimes be insufficient to identify an individual (20). Therefore, analysis of the mtDNA control region is performed, as it facilitates species identification (7). Additionally, the hypervariable regions of the mtDNA D-loop are used for identification $(1,4,15)$. Verscheure et al. $(18,19)$ used dogs' informative sites of the mtDNA control region to improve discrimination power in forensic casework. They confirmed their suitability for enhancing dog mtDNA discrimination power and increase the exclusion probability of forensic dog mtDNA analysis.

The use of the mtDNA of the domestic dog in criminal and civil proceedings was introduced in the 1990s (15). However, due to the weak connection between a specific mtDNA haplotype of a dog and its breed, such analyses are employed mainly to exclude an individual rather than achieve a positive identification thereof $(2,4)$.
The complete nucleotide sequence of the mitochondrial genome of the domestic dog Canis lupus familiaris was first described by Kim et al. (11). The mtDNA molecule contains 16727 base pairs (bp) and encodes 37 genes. The non-coding sequences of the mitochondrial genome constitute its small part, while the control region (CR) with a length of $1270 \mathrm{bp}$ devoid of genes is its largest fragment (11). The control region is located between $15458 \mathrm{bp}$ and $16727 \mathrm{bp}$ (1270 bp). It contains hypervariable regions HVI (15 458-16 130) and HVII (16 430-16 727), between which there is a VNTR (variable number of tandem repeats) region characterized by a variable number of tandem repeats of a 10-nucleotide motif (5'-GTACACGT(A/G)C-3'). These repeats are located between $16130 \mathrm{bp}$ and $16430 \mathrm{bp}(12,17)$. Due to its high mutagenicity, the region between nucleotides 15595 and 15653 in HVI is known as a "hotspot." The mutagenic potential of $\mathrm{CR}$ and the variable number of repeats in the VNTR region differentiate the mtDNA of each individual; hence, analysis thereof can be helpful in identification of individuals (20).

Polymorphisms in the HVI region of the mtDNA of the domestic dog were detected for the first time in 1997 (14), when the presence of 22 point mutations in the mtDNA of 52 dog breeds was reported (14). 
In 2007, mtDNA sequences from 43 dog breeds were analyzed showing 21 SNPs (single nucleotide polymorphisms) (8). In 2009, 96 SNP mutations were identified (20). Sugiyama et al. (16) analyzed mtDNA sequences in pedigree dogs from Japan. Forty-seven SNP mutations were detected. On the other hand, the results of mtDNA analysis of different dog breeds published in 2017 showed the presence of 22 SNPs (6).

Thus far, the application of canine mtDNA analysis for identification of individuals has not been exhaustively investigated. Given the growing need for medical-legal identification of genetic material originating from dogs, efforts continue to determine individual- or breed-specific SNPs in mtDNA. Therefore, the aim of the study was to determine molecular differences between different breeds of dogs by comparative analysis of the mitochondrial DNA D-loop sequence.

\section{Material and methods}

The analyses were carried out on blood collected in veterinary clinics from 40 dogs: 17 Doberman Pinscher dogs, 5 Dachshund dogs, 11 German Shepherd dogs, and 7 Crossbreed dogs. DNA was isolated with the DNeasy Blood and Tissue Kit (Qiagen, Hilden, Germany). The genetic material was assessed qualitatively (electrophoretic separation in agarose gel) and quantitatively (BioPhotometer spectrophotometer; Eppendorf, Hamburg, Germany).

Amplification of D-loop fragments was performed by the DNA polymerase chain reaction (PCR) technique in a Labocycler Thermal Cycler (Sensoquest Biomedical). The temperature-time profile of the reaction and the composition of the reaction mixture for the D-loop fragment were determined experimentally. Primers used in the analysis were found in literature reports (2); they were contained between nucleotides 15341 and 15804 (LF (5'-TTACCTTGGTCTTGTAAACC) and HR (5'-CTGAAGTAAGAACCAGATGCC).

The PCR products were visualized in $2 \%$ agarose gel. Amplicons were sequenced using a BigDye Terminator Cycle Sequencing kit (Applied Biosystem, USA) in the GeneAmp PCR system 9700 (Applied Biosystem, USA). The samples were subsequently purified on CentriSep columns according to the manufacturer's protocol or precipitated with ethanol and sodium acetate according to the protocol of the BigDye kit manufacturer. Extension products were separated on an ABI 377 automated sequencer (Applied Biosystem, USA).

The amplicons were analyzed to detect the sites of polymorphisms within mtDNA fragments from each animal (DNA BaserSequence Assembler v4 (2013); MEGA v 6.06 (2014); Chromas v 2.1.1 (2012)). The nucleotide sequences of the D-loop in each breed of dogs were compared with a reference sequence (GenBank accession No. U96639, 11).
The study was approved by the Second Local Ethical Committee for Animal Experiments in Lublin, Poland (approval number 7/2013).

\section{Results and discussion}

Polymorphic positions. Sequences of the HVI region of mtDNA from 40 Canis lupus familiaris individuals of different breeds were analyzed. Fifteen SNP (single nucleotide polymorphism) mutations were detected, including 14 substitution polymorphisms with transitions constituting a vast majority (93\%). In addition, a transversion mutation in position m.T15639G/A and insertion in position m.15464insC were found (Tab. 1).

On the basis of the frequency of SNPs, hot spots were identified in positions m.15526, m.15595, m.15612, m.15632, m.15639, m.165643, and m.16652 (Tab. 1). The most frequent change was a transition in position m.15639 (90\%), i.e. m.T15639G (54\%) and m.T15639A (36\%).

Intrabreed analysis of SNPs. Due to the frequency of point mutations in the control region of the canine mtDNA D-loop, no breed-specific polymorphisms were detected. Therefore, an attempt was made to determine SNP patterns that could indicate the breed affiliation of individual dogs.

The identified SNPs were compiled for each breed of the dogs analyzed. The analysis of the HVI region of mtDNA revealed two SNP patterns in $94 \%$ of the Doberman Pinscher dogs (Tab. 2). These SNP patterns were absent in only one Doberman dog, in

Tab. 1. Polymorphisms observed in the HV-I region of mtDNA between the reference sequence and the analyzed sequences within breeds

\begin{tabular}{|c|c|c|c|c|c|}
\hline \multicolumn{6}{|c|}{ Prevalence in dog breed [\%] } \\
\hline $\begin{array}{l}\text { Reference } \\
\text { sequence }\end{array}$ & $\begin{array}{l}\text { Sequence } \\
\text { in dog blood }\end{array}$ & $\begin{array}{c}\text { Doberman } \\
\text { Pinscher }\end{array}$ & Dachshund & $\begin{array}{c}\text { German } \\
\text { Shepherd }\end{array}$ & Crossbreed \\
\hline m.15464_15465 & m.155464.1C & - & - & - & 14 \\
\hline m.15475T & m.T15475C & - & - & 45 & - \\
\hline m.15508C & m.C15508T & 29 & - & - & 14 \\
\hline m.15526C* & m.C15526T & 94 & 20 & - & 57 \\
\hline m.15595C* & m.C15595T & 65 & 20 & - & 29 \\
\hline m.15611T & m.T15611C & 29 & - & - & 14 \\
\hline m.15612T* & m.T15612C & 65 & 20 & - & 43 \\
\hline m.15620T & m.T15620C & 6 & 20 & 27 & - \\
\hline m.15621C & m.C15621T & 29 & - & - & - \\
\hline m.15627A & m.A15627G & 6 & 40 & 73 & - \\
\hline m.15632C* & m.C15632T & 65 & 40 & 45 & 43 \\
\hline m.15639T* & $\begin{array}{l}\text { m.T15639G } \\
\text { m.T15639A }\end{array}$ & $\begin{array}{r}94 \\
6\end{array}$ & $\begin{array}{l}20 \\
80\end{array}$ & 82 & $\begin{array}{l}57 \\
14\end{array}$ \\
\hline m.15643A* & m.A15643G & 65 & - & - & 43 \\
\hline m.15650T & m.T15650C & 29 & - & - & 14 \\
\hline m.15652G* & m.G15652A & 65 & 20 & - & 43 \\
\hline
\end{tabular}

Explanation: positions in the reference sequence of mtDNA marked * - mean highly informative SNPs in the present study 
Tab. 2. Patterns of SNPs in the HVI region of mtDNA in the Doberman Pinscher

\begin{tabular}{|c|c|c|}
\hline $\begin{array}{l}\text { Position in reference } \\
\text { sequence }\end{array}$ & SNP7* & SNP6* \\
\hline m.15508C & m.C15508 & m.C15508T* \\
\hline m.15526C & m.T15526T* & m.T15526T* \\
\hline m.15595C & m.C15595T* & m.C15595 \\
\hline m.15611T & m.T15611 & m.T15611C* \\
\hline m.15612T & m.T15612C* & m.T15612 \\
\hline m.15621C & m.C15621 & m.C15621T* \\
\hline m.15632C & m.C15632T* & m.C15632 \\
\hline m.15639T & m.T15639G* & m.T15639G* \\
\hline $\mathrm{m} .15643 A$ & m.A15643G* & m.A15643 \\
\hline m.15650T & m.T15650 & m.T15650C* \\
\hline $\mathrm{m} .15652 \mathrm{G}$ & m.G15652A* & m.G15652 \\
\hline
\end{tabular}

Explanation: * - The pattern of seven (SNP7) and six (SNP6) polymorphisms observed in the Doberman Pinscher

Tab. 3. Pattern of SNPs in the HVI region of mtDNA in the German Shepherd

\begin{tabular}{|c|c|c|}
\hline $\begin{array}{l}\text { Position in reference } \\
\text { sequence }\end{array}$ & SNP4* & SNP3* \\
\hline m.15475T & m.T15475C* & m.T15475 \\
\hline m.15620T & m.T15620 & m.T15620C* \\
\hline m.15627A & m.A15627G* & m.A15627G* \\
\hline m.15632C & m.C15632T* & m.C15632 \\
\hline m.15639T & m.T15639A* & m.T15639A* \\
\hline
\end{tabular}

Explanation: * - The pattern of four (SNP4) and three (SNP3) polymorphisms observed in the German Shepherd

which 3 SNPs in positions m.15620, m.15627, and m.15639 were identified. The polymorphisms are shown in Table 2 as SNP7 and SNP6 patterns. The former polymorphism pattern (SNP7) was observed in $69 \%$ of the Doberman dogs, whereas the latter (SNP6) was characteristic of the other $31 \%$ of the dogs of this breed. Only two SNPs, i.e. m.C15526T and m.T15639G, were noted in the analyzed sequences from all individuals.

Specific polymorphism patterns were also observed in sequences from the German Shepherd dogs. Pattern SNP4 was detected in $45.5 \%$ of the dogs, whereas $27 \%$ of the animals exhibited 3 other polymorphisms (SNP3) (Tab. 3). Noteworthy, the m.T15475C SNP was detected only in those German Shepherd dogs in which 3 other mutations were detected, together forming the SNP4 pattern.

The Dachshund breed was characterized by the lowest number of mutations. No SNPs that could be regarded as specific for this breed were identified. The analysis of the genetic material from the Crossbreed dogs showed the greatest diversity. There were sequences without SNPs in the region analyzed and various sporadic mutations, e.g. m.15464.1C insertion (Tab. 1).
Given the condition of material collected for examinations, the only solution is often to analyze mtDNA, since it can be amplified even if there are only small amounts of DNA or it is degraded (9). Although a greater emphasis is placed on the analysis of mtDNA from human material, canine mtDNA has occasionally been analysed in criminal and civil proceedings $(1,2$, $13,15)$. To date, the results of analyses of the control region of the mtDNA D-loop from domestic dogs have been reported from such countries as Austria (5), Japan (16), Sweden (14), Turkey (6), and the United States (8). However, there has been only one report on the use of mtDNA for identification of dogs originating from Poland (4).

The SNPs in the HVI region presented in this study occur frequently and have already been reported by other authors $(6,8,10,16,20)$. The analysis revealed 15 polymorphisms in the HVI region of the mtDNA D-loop (Tab. 1).

The number of SNPs detected in this region by other authors varies. Savolein et al. (13) identified 22 SNPs in an analysis of hair from 102 dogs, Eichman and Parson (5) noted 40 polymorphisms in mtDNA from 133 dogs, and Erkan et al. (6) identified 27 mutations in material collected from 150 dogs. The present results revealed point mutations (Tab. 1), some of which were located in the region between nucleotides 15595 and 15 653, which is referred to as a "hotspot" with high mutagenic potential $(8,9)$.

The most common type among the SNPs identified in this study was a transition in position m.15639 (90\%). This substitution has frequently been reported. The results presented in Table 1 are consistent with those obtained by Eichmann and Parson (5), who detected a polymorphism in position $\mathrm{m} .15639$ in $87.5 \%$ of dogs, where the replacement of thymine with guanine accounted for $25 \%$ of cases, while adenine replaced thymine in $62.5 \%$ of cases. Erkan et al. (6) found this mutation, including m.T15639G $(12.7 \%)$ and m.T15639A (32.5\%), in $45.2 \%$ of dogs.

Interesting results were obtained by analysing the hypervariable HVI region in the material from the Doberman Pinscher and German Shepherd dogs (Tab. 2 and 3). The analysis of polymorphisms revealed the existence of two sequence patterns in most dogs of these breeds (94\% of Doberman dogs, 73\% of German Shepherd dogs). Breed-specific patterns of polymorphisms are reported here for the first time in the literature. Other authors usually indicate mutations in the material without reference to the dog breed (6) or identify haplotypes on the basis of sequences obtained $(9,16)$. Moreover, sequences for analysis frequently originate from only one (14) or a few (9) members of a breed, which prevents identification of breed-specific polymorphism patterns.

To date, the relationship between the dog breed and the occurrence of individual mutations has been addressed in only few papers. Gundry et al. (8) at- 
tempted to identify characteristic mutations in Golden Retriever and Labrador Retriever dogs. However, due to the high frequency of mutations in both breeds, it was impossible to determine their breed specificity. There are no literature reports on characteristic mutations in Doberman and German Shepherd dogs; hence, it is impossible to compare the present results with literature data.

The current knowledge about genetic identification based on the analysis of the mitochondrial DNA of the domestic dog is insufficient to determine breedspecific SNPs. Therefore, it is necessary to continue these investigations in different dog breeds.

Investigations have confirmed the presence of polymorphisms in the HVI region of mtDNA in various dog breeds described in the literature. However, the present study proposes a new solution for dog breed identification, namely, the application of breed-specific SNP patterns. The analysis revealed the presence of already known SNPs in various configurations depending on the breed. Two SNP patterns specific for Doberman and German Shepherd dogs were identified. They were detected in most dogs of these breeds (in 94\% of Doberman dogs and in 73\% of German Shepherd dogs). Determination of breed-specific SNP patterns, together with estimation of the probability of their presence in a given breed, may facilitate accurate identification of material from the domestic dog in the future.

\section{References}

1. Aaspõllu A., Kelve M.: The first criminal case in Estonia with dog's DNA data admitted as evidence. Int. Congr. Ser. 2003, 1239, 847-851.

2. Asch B. van, Albarra C., Alons A., Angu R., Alve C., Betanco E., Catanes C. I., Corac D., Crespill M., Doutremepuic C., Estonb A., Fernande A. T., Fernande E., Garci A. M., Garci M. A., Gilard P., Goncalve R., Hernande A., Lim G., Nasciment E., de Pancorb M. M., Parr D., Pinheiro Mde F., Prat E., Puente J., Ramirez J. L., Rendo F., Rey I., Di Rocco F., Rodriguez A., Sala A., Salla J., Sanchez J. J., Sola D., Silva S., Pestano Brito J. J., Amorim A.: Forensic analysis of $\operatorname{dog}$ (Canis lupus familiaris) mitochondrial DNA sequences: an inter-laboratory study of the GEP-ISFG working group. Forensic Sci. Int.-Gen. 2009, 4, 49-54.

3. Barrientos L. S., Crespi J. A., Fameli A., Posik D. M., Morales H., Peral Garcia P., Giovambattista G.: DNA profile of dog feces as evidence to solve a homicide. Leg. Med. 2016, 22, 54-57.

4. Branicki W., Kupiec T., Pawłowski R.: Analysis of dog mitochondrial DNA for forensic identification purposes. Probl. Forensic Sci. 2002, 50, 91-98.

5. Eichmann C., Parson W.: Molecular characterization of the canine mitochondrial DNA control region for forensic applications. Int. J. Legal Med. 2007, 121, 411-416.

6. Erkan I., Dastan K., Karadeniz S., Yıldız Y., Alpsar D., Yukseloglu E. Mitochondrial DNA analysis of the domestic dogs in Turkey. Europ. J. Forensic Sci. $2017,4,1$

7. Fumagalli L., Cabrita C. J., Castella V.: Simultaneous identification of multiple mammalian species from mixed forensic samples based on mtDNA control region length polymorphism. Forensic Sci. Int. 2009, 2, 302-303.

8. Gundry R. L., Allard M. W., Moretti T. R., Honeycutt R. L., Wilson M. R., Monson K. L., Foran D. R.: Mitochondrial DNA analysis of the domestic dog: control region variation within and among breeds. J. Forensic Sci. 2007, 52, 562-572.

9. Himmelberger A. L., Spear T. F., Satkoski J. A., George D. A., Garnica W. T., Malladi V.S., Smith D. G., Webb K. M., Allard M. W., Kanthaswamy S.: Forensic Utility of the Mitochondrial Hypervariable Region 1 of Domestic Dogs, in Conjunction with Breed and Geographic Information. J. Forensic Sci. 2008, 53, 81-89.

10. Imes D. L., Wictum E. J., Allard M. W., Sacks B. N.: Identification of single nucleotide polymorphisms within the mtDNA genome of the domestic dog to discriminate individuals with common HVI haplotypes. Forensic Sci. Int. Genet. 2012, 6, 630-639.

11. Kim K. S., Lee S. E., Jeong H. W., Ha J. H.: The Complete Nucleotide Sequence of the Domestic Dog (Canis familiaris) Mitochondrial Genome. Mol. Phylogenet. Evol. 1998, 10, 210-220.

12. Kowal K., Ślaska B., Bownik A., Horecka B., Gawor J., Śmiech A., Tkaczyk A. Analysis of Mitochondrial Genome from Labrador (Canis lupus familiaris) with Mammary Gland Tumour Reveals Novel Mutations and Polymorphisms. Ann. Anim. Sci. 2019, 19, 619-632.

13. Savolainen P., Lundeberg J.: Forensic Evidence Based on mtDNA from Dog and Wolf Hairs. J. Forensic Sci. 1999, 44, 77-81.

14. Savolainen P., Rosen B., Holmberg A., Leitner T., Uhlgn M., Lundeberg J.: Sequence Analysis of Domestic Dog Mitochondrial DNA for Forensic Use. J. Forensic Sci. 1997, 42, 593-600.

15. Schneider P. M., Seo Y., Rittner C.: Forensic mtDNA hair analysis excludes a dog from having caused a traffic accident. Int. J. Legal Med. 1999, 112, 315-316.

16. Sugiyama S., Chong Y. H., Shito M., Kasuga M., Kawakami T., Udagawa C., Aoki H., Bonkobara M., Tsuchida S., Sakamoto A., Okuda H., Nagai A., Omi T. Analysis of mitochondrial DNA HVR1 haplotype of pure-bred domestic dogs in Japan. Legal Med.-Tokyo 2013, 15, 303-309.

17. Ślaska B., Surdyka M., Brodzki A., Nisztuk S., Gurgul A., Bugno-Poniewierska M., Śmiech A., Różańska D., Orzelski M.: Mitochondrial D-loop mutations can be detected in sporadic malignant tumours in dogs. B. Vet. I. Pulawy 2014, 58, 631-637.

18. Verscheure S., Backeljau T., Desmyter S.: Coding region SNP analysis to enhance dog mtDNA discrimination power in forensic casework. Forensic Sci. Int.-Gen. 2015, 14, 86-95.

19. Verscheure S., Backeljau T., Desmyter S.: Dog mitochondrial genome sequencing to enhance dog mtDNA discrimination power in forensic casework. Forensic Sci. Int.-Gen. 2014, 12, 60-68.

20. Webb K. M., Allard M. W.: Identification of forensically informative SNPs in the domestic dog mitochondrial control region. J. Forensic Sci. 2009, 54, 289-304

Corresponding author: prof. dr hab. Brygida Ślaska, DVM, PhD, Akademicka 13, 20-950 Lublin, Poland; e-mail: brygida.slaska@up.lublin.pl 